(2) Open Access Full Text Article

\title{
Impact of the COVID-19 Pandemic on the Management of Acute Myocardial Infarction
}

\author{
Jing Han* \\ Ruofei Jia* \\ Chengzhi Yang* \\ Zening Jin
}

Department of Cardiology and Macrovascular Disease, Beijing Tiantan Hospital, Capital Medical University, Beijing, People's Republic of China

*These authors contributed equally to this work
Correspondence: Zening Jin Department of Cardiology and Macrovascular Disease, Beijing Tiantan Hospital, No. 119 South Fourth Ring West Road, Beijing, 100070, People's Republic of China

Email jin_zening@ccmu.edu.cn
Aim: The coronavirus (COVID-19) outbreak in 2019 has negatively impacted the care of patients with other life-threatening diseases, including acute myocardial infarction (AMI). However, there is little published information concerning the depth of the impact on the clinical management and outcome following AMI.

Methods: We enrolled patients with AMI who received urgent primary percutaneous coronary intervention at the Beijing Tiantan Hospital (Beijing, China) between December 1, 2019 and April 10, 2020. Patients were divided into 2 cohorts, the preCOVID-19 group (from December 1, 2019 to January 31, 2020) and during-COVID-19 group (from February 1, 2020 to April 10, 2020) for analysis. The door-to-balloon (D to B) time, total hospitalization stay (days) and coronary care unit (CCU) hospitalization days were calculated. New York Heart Association heart functional class (NYHA class), rehospitalization and death ratio in patients were assessed between the two cohorts.

Results: A total of 148 AMI patients were enrolled in this study comprising 53 patients preCOVID-19 group and 95 patients during-COVID-19 group. Patients with AMI duringCOVID-19 group had longer symptom onset to hospital time (4.5 [2.0-9.3] vs 3.0 [2.05.0] hours, $\mathrm{p}=0.013$ ) and $\mathrm{D}$ to $\mathrm{B}$ time (96 [74-119] vs 67 [52-81] minutes, $\mathrm{p}<0.001$ ); the $\mathrm{D}$ to $\mathrm{B}$ time shortened during the study period. The two cohorts did not have significantly different number of hospitalization days, re-hospitalization rates, peak cTnI, BNP or death rates. For the one-year follow-up, the patients in the during-COVID-19 group were classified as NYHA class III-IV more frequently $(9[9.7 \%]$ vs 0 [0\%], p=0.004).

Conclusion: The COVID-19 pandemic significantly affected one measure of critical care of patients with AMI, NYHA classification, which may have resulted in increased medical expenses.

Keywords: acute myocardial infarction, COVID-19 pandemic, in-hospital outcome, New York Heart Association heart functional class

\section{Introduction}

The 2019 coronavirus (COVID-19) has rapidly spread worldwide and infected millions of people. To combat further COVID-19 infection and mortality, response tactics have been established and include quarantine and social distancing. ${ }^{1-4}$ While these measures have helped to prevent the spread of COVID-19, the medical care of patients with other diseases such as AMI has been affected.

Garcia et al recently showed there was a $38 \%$ reduction in ST-elevation myocardial infarction (STEMI) cardiac catheterization laboratory activations due to COVID-19. ${ }^{5}$ Thus, reperfusion therapy for AMI patients may currently be insufficient during the COVID-19 pandemic. As an early epicenter of COVID-19, China 
rigidly enforced social distancing and quarantine to effectively control COVID-19 spread. Concurrently, hospitals suspended selective operations, including percutaneous coronary intervention (PCI) due to COVID-19, but STEMI as the first treatment strategy was continued, but managed in a different way. Because regional emergency medical system (EMS)-based STEMI networks were closed, thrombolysis was chosen as the first-line therapy in most hospitals in Beijing, while primary PCI was still performed for patients with STEMI and non-STEMI when justified, and following COVID-19 screening in patients.

Currently, there is little information on the direct impact of COVID-19 on clinical management and outcomes of AMI. The current study aimed to investigate the procedural characteristic utilized in our hospital during the COVID-19 crisis to measure in-hospital outcomes and one-year follow-up outcomes of AMI in patients.

\section{Methods}

\section{Study Population and Design}

The present study was approved by the Ethics Committee of Beijing Tiantan Hospital, Capital Medical University (Beijing, China), and performed according to the Declaration of Helsinki. This is a retrospective observational study or archived data, thus informed consent was unnecessary. Our study used numbers instead of names to identify participants. Removed that contain identifiers, such as names and addresses. The data was accessible only to researchers and was not leaked. We retrospectively enrolled patients with AMI who received urgent primary percutaneous coronary intervention (PPCI) at the Beijing Tiantan Hospital from December 1, 2019 to April 10, 2020. The diagnosis of AMI was made according to combination of chest pain symptoms, electrocardiographic ST-T changes, atherosclerotic risk factors, and elevated cardiac troponin I at initial or subsequent testing. Patients were divided into pre- and during-COVID-19 cohorts. The pre-COVID-19 cohort consisted of 53 patients enrolled between December 1, 2019 and January 31, 2020, when standardized healthcare was not influenced by the COVID19. Starting on February 1, 2020, when social distancing was effective, all patients were screened for COVID-19 before the emergency coronary angiography. Screening included a history of close contact with patients from the pandemic area, body temperature, blood count, chest computerized tomography (CT) imaging, and DNA specimens were collected to assay for severe acute respiratory syndrome coronavirus 2 (SARS-CoV2). We reserved a specific CT equipment for these patients. Because the whole numbers of infected patients were low in Beijing, if the chest CT imaging result was normal, we would performed the PPCI without waited for the nucleic acid result. A total of 95 patients were enrolled in the during-COVID -19 cohort in 10 weeks.

\section{Data Collection}

Evaluation of patients included complete medical history, physical examination, blood examination, transthoracic echocardiography, and coronary angiography. The symptom onset to hospital time, body length, weight, blood pressure and heart rate on admission, atherosclerotic risk factors, reports of laboratory measurements, echocardiography and coronary angiography were documented. Symptom onset to hospital time was defined as the time from patient-reported chest discomfort onset to the time of arrival at Accident and Emergency Department of Beijing Tiantan Hospital. The door-to-balloon (D to B) time, total hospitalization days, CCU hospitalization days and oneyear follow-up outcomes were calculated.

\section{Statistical Analysis}

Continuous variables are expressed as mean $\pm \mathrm{SD}$ or median (interquartile range [IQR]), according to their normality, and categorical variables are shown as frequencies (percentages). Comparisons of continuous variables between two groups were assessed using independent Student's $t$-test or Mann-Whitney $U$-test depending on the distribution of variables. One-way ANOVA was used to compare continuous variables more than two groups. The chi-square test was used for comparisons between categorical variables, and Fisher's exact test was used when expected frequency was $<5$. A 2-tailed $p$ value $<0.05$ was considered as statistically significant. Statistical analysis was performed with SPSS version 19.0 software (SPSS Inc, Chicago, Illinois).

\section{Results}

Baseline characteristics of the patients with AMI are summarized in Table 1. The age, sex and atherosclerotic risk factors were not significantly different between the two cohorts. Compared with AMI patients pre- COVID-19, patients during-COVID-19 had significant higher C-reactive protein (CRP) levels. However, there was no significant difference in total leucocyte count or neutrophil 
Table I Baseline Characteristics of Patients with Acute Myocardial Infarction Pre- and During-COVID-19

\begin{tabular}{|c|c|c|c|}
\hline Clinical Characteristics & Pre-COVID-19 $(n=53)$ & During-COVID-I9 (n = 95) & $P$ value \\
\hline Age (years) & $59.3 \pm 12.2$ & $59.8 \pm 11.9$ & 0.802 \\
\hline Male, no. (\%) & $4 \mathrm{I}(77.4 \%)$ & 77 (8I.1\%) & 0.671 \\
\hline BMI $\left(\mathrm{kg} / \mathrm{m}^{2}\right)$ & $28.7 \pm 7.6$ & $29.1 \pm 8.5$ & 0.776 \\
\hline \multicolumn{4}{|l|}{ Risk factors, no. (\%) } \\
\hline Coronary artery disease & $9(17.0 \%)$ & $12(12.6 \%)$ & $0.47 I$ \\
\hline Hyperlipidemia & II (20.8\%) & 29 (30.5\%) & 0.248 \\
\hline Diabetes mellitus & $15(28.3 \%)$ & $26(27.4 \%)$ & 1.000 \\
\hline Hypertension & $28(52.8 \%)$ & $5 \mathrm{I}(53.7 \%)$ & 1.000 \\
\hline Current smokers & $25(47.2 \%)$ & $54(56.8 \%)$ & 0.304 \\
\hline Stroke & $4(7.5 \%)$ & $13(13.7 \%)$ & 0.297 \\
\hline \multicolumn{4}{|l|}{ Laboratory examination } \\
\hline Total cholesterol (mmol/L) & $4.64 \pm 1.25$ & $4.56 \pm 0.99$ & 0.647 \\
\hline LDL cholesterol (mmol/L) & $3.04 \pm 1.10$ & $2.94 \pm 0.88$ & 0.558 \\
\hline HDL cholesterol (mmol/L) & $1.02 \pm 0.26$ & $1.04 \pm 0.23$ & 0.779 \\
\hline Leucocyte $\left(10^{\wedge 9 / L)}\right.$ & $11.69 \pm 9.61$ & $10.74 \pm 3.19$ & 0.384 \\
\hline Neutrophils (10^9/L) & $7.29 \pm 2.70$ & $7.99 \pm 3.12$ & 0.187 \\
\hline CRP (mg/L) & $1.52(0.44-3.39)$ & $3.37(1.09-10.4 I)$ & 0.002 \\
\hline Fast glucose $(\mathrm{mmol} / \mathrm{L})$ & $6.4 \mathrm{I} \pm 2.6 \mathrm{I}$ & $6.80 \pm 2.79$ & 0.410 \\
\hline Glycosylated hemoglobin (\%) & $6.66 \pm 1.81$ & $6.47 \pm 1.44$ & 0.507 \\
\hline Serum creatinine $(\mu \mathrm{mol} / \mathrm{L})$ & $74.98 \pm 21.50$ & $77.63 \pm 20.85$ & 0.466 \\
\hline eGFR (mL/min) & $100.80 \pm 21.54$ & $98.47 \pm 20.12$ & 0.511 \\
\hline Hemoglobin $(g / L)$ & $|47.59 \pm 15.7|$ & $145.73 \pm 17.32$ & 0.519 \\
\hline Albumin $(g / L)$ & $40.98 \pm 3.79$ & $40.55 \pm 3.95$ & 0.534 \\
\hline
\end{tabular}

Note: Data are expressed as mean $\pm \mathrm{SD}$, number (percentage), or median (interquartile range).

Abbreviations: BMI, body mass index; CRP, C-reactive protein; eGFR, estimated glomerular filtration rate; HDL, high density lipoprotein; LDL, low density lipoprotein.

count between two cohorts. No patients were confirmed with COVID-19 in our cohort.

\section{Procedural Characteristics of Patients with AMI Pre- and During-COVID-19}

As shown in Table 2, STEMI was very frequent in AMI patients both pre- and during-COVID-19. The time from symptom onset to hospital was significantly longer in patients during-COVID-19 than that in patients pre-COVID -19 (4.5 [2.0-9.3] vs 3.0 [2.0-5.0] hours, $p=0.013)$. Moreover, patients enrolled during-COVID-19 also had longer D to B time compared to the pre-COVID-19 group (96 [74-119] vs 67 [52-81] minutes, $p<0.001$ ). Interestingly, we found although the D to B time was markedly prolonged at the beginning of COVID-19 epidemic, and it became shorter in the later ten weeks (Figure 1A). However, there was no significant change in symptom onset to hospital time during the COVID-19 epidemic (Figure 1B).
Table 2 Procedural Characteristics of Patients with Acute Myocardial Infarction Pre- and During-COVID-19

\begin{tabular}{|c|c|c|c|}
\hline Variables & $\begin{array}{l}\text { Pre-COVID } \\
-19(n=53)\end{array}$ & $\begin{array}{l}\text { During- } \\
\text { COVID-19 } \\
(n=95)\end{array}$ & $P$ value \\
\hline STEMI & 47 (88.7\%) & 78 (82.1\%) & 0.350 \\
\hline $\begin{array}{l}\text { Symptom onset to } \\
\text { hospital time (hours) }\end{array}$ & $3.0(2.0-5.0)$ & $4.5(2.0-9.3)$ & 0.013 \\
\hline $\begin{array}{l}\text { Door-to-balloon } \\
\text { (D2B) time (minutes) }\end{array}$ & $67(52-8 I)$ & $96(74-119)$ & $<0.001$ \\
\hline $\begin{array}{l}\text { Infarct-related artery, } \\
\text { no. (\%) }\end{array}$ & & & 0.089 \\
\hline LAD & 32 (60.4\%) & $4 \mathrm{I}(43.2 \%)$ & \\
\hline LCX & $3(5.7 \%)$ & $12(12.8 \%)$ & \\
\hline RCA & I8 (34.0\%) & 42 (44.7\%) & \\
\hline
\end{tabular}

Note: Data are expressed as mean $\pm \mathrm{SD}$, number (percentage), or median (interquartile range).

Abbreviations: LAD, left anterior descending coronary artery; LCX, left circumflex coronary artery; RCA, right coronary artery; STEMI, ST-segment elevation myocardial infarction. 

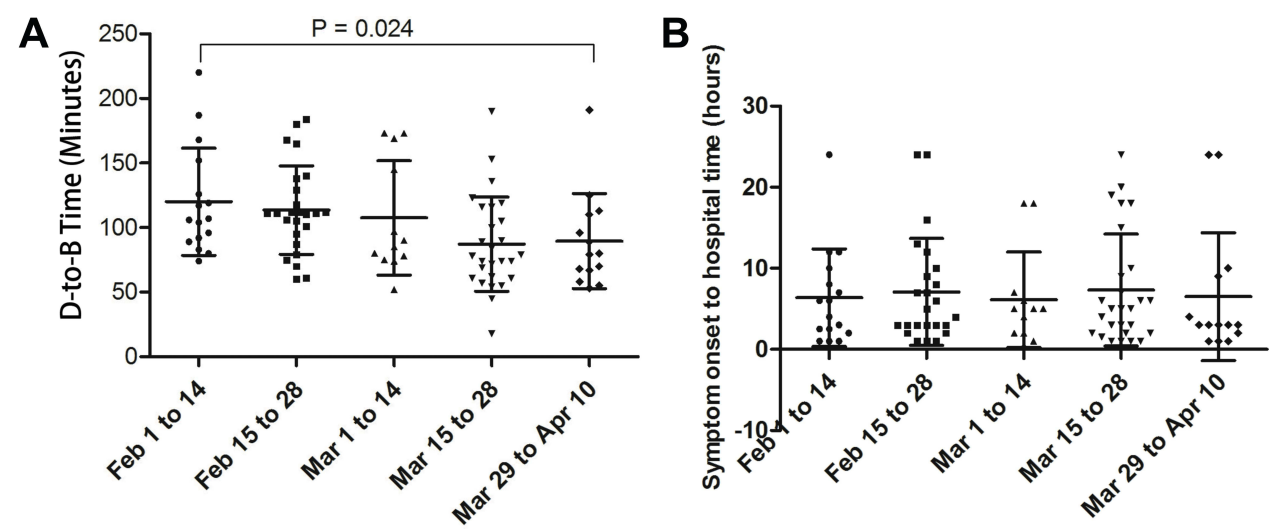

Figure I (A) Changes in door-to-balloon (D to B) time and (B) symptom onset to hospital time during the COVID-I9 pandemic.

There was a small difference in infarct-related artery type between the two cohorts: the left anterior descending coronary artery (LAD) was the most commonly affected vessel in patients pre-COVID-19, while the right coronary artery (RCA) was more common in patients during-COVID-19.

\section{Severity and In-Hospital Outcomes of AMI Pre- and During-COVID-19}

Patients with AMI pre- and during-COVID-19 did not have significantly different initial systolic blood pressure, CCU hospitalization days, total hospitalization days, death ratio, peak cTnI or BNP. However, the left ventricular ejection fraction (LVEF) was significantly higher in patients duringCOVID-19 than that in patients pre-COVID-19 (Table 3.).

\section{One Year Follow-Up Outcomes of AMI Pre-and During-COVID-19 Groups}

There was no difference in death ratio between pre- and during-COVID-19 groups (2[3.8\%] vs $2[2.1 \%], \mathrm{p}=0.550)$.
However, patients enrolled during-COVID-19 developed NYHA class III-IV more frequently $(9[9.7 \%]$ vs $0[0 \%]$, $\mathrm{p}=0.004)$ (Table 4).

\section{Discussion}

The present study revealed that there was no effect of hospitalization during COVID-19 on hospitalization days, peak cTnI, BNP or death ratio, but AMI patients had increased D to B time and symptom onset to hospital time. Most important is the observation that duringCOVID-19 more AMI patients were classified as NYHA III-IV more frequently at one-year follow-up.

Recent literature suggests that the COVID-19 pandemic delayed critical medical care and clinical management of AMI patients requiring emergency treatment. In a study with 7 STEMI patients, Tam et al showed that the symptom onset to hospital time, door-to-device time and catheterization laboratory arrival-to-device time were prolonged after COVID-19 breakout, $^{6}$ and Hammad et al

Table 3 In-Hospital Outcomes of Acute Myocardial Infarction Pre- and During-COVID-19

\begin{tabular}{|l|c|c|c|}
\hline Variables & Pre-COVID-I9 (n = 53) & During-COVID-19 (n = 95) & P value \\
\hline Systolic blood pressure (mmHg) & $127.6 \pm 20.5$ & $123.4 \pm 23.8$ & 0.282 \\
CCU hospitalization days & $3.54 \pm 3.25$ & $3.73 \pm 2.91$ & 0.724 \\
Total hospitalization days & $8.76 \pm 4.79$ & $9.35 \pm 5.80$ & 0.539 \\
Killip functional class III or IV, no. (\%) & $9(17.0 \%)$ & $15(15.8 \%)$ & 1.000 \\
Shock, no. (\%) & $16(30.2 \%)$ & $19(20.0 \%)$ & 0.226 \\
Death, no. (\%) & $2(3.8 \%)$ & $2(2.1 \%)$ & 0.620 \\
Lowest level LVEF (\%) & $53.0 \pm 8.3$ & $56.0 \pm 7.8$ & 0.034 \\
Peak cTnl & $66.8(23.3-139.5)$ & $46.7(19.1-117.0)$ & 0.289 \\
Peak BNP & $347.6(232.0-763.7)$ & $361.9(198.3-701.8)$ & 0.489 \\
Peak CRP & $12.4(6.8-61.6)$ & $22.0(7.6-70.0)$ & 0.409 \\
\hline
\end{tabular}

Note: Data are expressed as mean \pm SD, number (percentage), or median (interquartile range).

Abbreviations: BNP, B-type natriuretic peptide; CCU, coronary care unit; CRP, C-reactive protein; cTnl, cardiac troponin I; LVEF, left ventricular ejection fraction. 
Table 4 Outcomes of Acute Myocardial Infarction Pre- and During-COVID-19 at I-Year Follow-Up

\begin{tabular}{|l|c|c|c|}
\hline Variables & $\begin{array}{c}\text { Pre-COVID } \\
-19(\mathbf{n}=\mathbf{5 3})\end{array}$ & $\begin{array}{c}\text { During- } \\
\text { COVID- I9 } \\
\text { (n = 95) }\end{array}$ & P value \\
\hline Death, no. (\%) & $2(3.8 \%)$ & $2(2.1 \%)$ & 0.550 \\
\hline NYHA heart functional & & & 0.004 \\
class, no. (\%) & $4 I(80.4 \%)$ & $76(81.7 \%)$ & \\
I & $10(19.6 \%)$ & $8(8.6 \%)$ & \\
II & 0 & $9(9.7 \%)$ & \\
III & 0 & 0 & \\
IV & & \\
\hline
\end{tabular}

Note: NYHA heart functional class and heart functional were assessed in 51 survivors of Pre-COVID-19 cohort and 93 survivors of During-COVID-19 cohort. Abbreviation: NYHA, New York Heart Association.

found there were more late-presenting STEMI patients during the COVID-19 pandemic. $^{7}$

The China Chest Pain Center reported national data in November, 2020, based upon 28,189 STEMI patients admitted to 1,372 Chest Pain Centers between December 27, 2019 and February 20, 2020. The results showed the COVID-19 outbreak reduced the number of STEMI cases, and the percentage of patients undergoing PPCI declined, while the percentage of patients undergoing thrombolysis increased. With an average delay of approximately $20 \mathrm{~min}$ for reperfusion therapy, the rate of in-hospital mortality and in-hospital heart failure increased during the outbreak, but the rate of in-hospital hemorrhage remained stable. ${ }^{8}$ Consistent with the previous studies, our data suggests that both symptom onset to hospital time and $\mathrm{D}$ to $\mathrm{B}$ time were delayed during COVID-19 pandemic. In our study, COVID-19 screening was performed before emergency coronary angiography, which often delayed angiography by 30 minutes. Therefore, it was not surprising that $\mathrm{D}$ to $\mathrm{B}$ time was markedly prolonged. Furthermore, because additional personal protective equipment (PPE) was necessary at all stages of treatment, there were additional delays incurred.

Of importance to the adequate treatment of AMI patients, we found that although the D to B time was increased at the beginning of COVID-19 epidemic, it was shortened later during the epidemic due to gradually improved screening and management algorithm. ${ }^{9}$ In contrast, the symptom onset to hospital time during the COVID-19 pandemic did not change significantly.

In Europe, especially, data from Italy, the second country touched by the epidemic after China from a temporal point of view, provided the similar and different results and other methods balanced epidemic and AMI patients. Firstly, several studies reported STEMI patients had a longer time from symptom onset to hospital presentation. In Rome, De Rosa et al observed a $48.4 \%$ reduction in admissions for AMI compared with the equivalent week in 2019. They summarized that admissions for AMI were significantly reduced during the COVID-19 pandemic across Italy, with a parallel increase in fatality and complication rates. ${ }^{10}$ In Milan, Cosentino et al observed a twofold longer time from symptom onset to hospital presentation and a three-fold higher rate of cardiogenic shock and in-hospital cardiac mortality during the COVID-19 outbreak when compared with those during the same time interval in $2019 .{ }^{11}$ They also provided a flowchart for shared early experience in AMI patients during epidemic. ${ }^{12}$ In our study, we had a similar result prolonged time from symptom onset to hospital and different result that the mortality remained at the same level.

To analyze the results, the Italian government centralized the treatment of STEMI in a very limited number of centres, which could control the epidemic more effectively, but might increase the difficulty for AMI patients in hospital presentation and cause panic among people. In Beijing, due to rapid and effective outbreak control of government, all the hospitals could receive AMI patients and perform PPCI. Because the whole city had a very low infected rate, each hospital could achieve the routine therapy with a limited influence by epidemic.

The key issue of importance in this study was to determine whether the COVID-19 pandemic would worsen the prognosis and treatment of AMI. Treatment delays are a very important index of the quality of care for an AMI patient. Hammad et al studied 35 STEMI patients during COVID-19 pandemic and found late-presenting patients had significant reduced LVEF and elevated troponin. ${ }^{7}$ However, our investigation showed that the COVID-19 pandemic did not affect peak troponin I, BNP, total hospitalization days, or in-hospital death ratio. The CCU hospitalization days were also not increased though some patients had to wait for nucleic acid test result before being transferred to general wards. The during-COVID-19 patients in our study had the higher LVEF, and this cohort of patients also had worse NYHA heart functional class. This suggests the possibility of rehospitalization and increased medical expenses for those patients, but that remains to be established. 


\section{Conclusions}

The COVID-19 pandemic negatively affected the medical care and clinical management of AMI patients. While we show that optimizing patient screening can help shorten inhospital delays, the COVID-19 pandemic did not affect inhospital outcomes, but it had a serious effect on NYHA heart functional class of patients. There are some limitations to this retrospective study that need to be stated. The study is a single-center study and the sample size was not very large due to limited enrollment time. A larger population and multi-center study may be needed in the future to further understand the impact of COVID-19 on the management of AMI patients and their quality of life.

\section{Acknowledgment}

We thank all healthcare workers who have sacrificed themselves in the COVID-19 pandemic.

\section{Funding}

This work was supported by the National Natural Science Foundation of China (grant 81900452).

\section{Disclosure}

The authors have no conflicts of interest to declare.

\section{References}

1. Yang C, Jin Z. An acute respiratory infection runs into the most common noncommunicable epidemic-COVID-19 and cardiovascular diseases. JAMA Cardiol. 2020;5(7):743. doi:10.1001/jamacardio.2020.0934

2. Li L, Hill J, Spratt JC, Jin Z. Myocardial injury in severe COVID-19: identification and management. Resuscitation. 2021;160:16-17. PMID: 33450334; PMCID: PMC7836289. doi:10.1016/j.resuscitation. 2020.11.040

3. Li Z, Guan X, Mao N, et al. Antibody seroprevalence in the epicenter Wuhan, Hubei, and six selected provinces after containment of the first epidemic wave of COVID-19 in China. Lancet Reg Health West Pac. 2021;8:100094. PMID: 33585828; PMCID: PMC7864613. doi:10.1016/j. lanwpc.2021.100094
4. Wu Z, McGoogan JM. Characteristics of and important lessons from the Coronavirus Disease 2019 (COVID-19) outbreak in China: summary of a report of 72314 cases from the Chinese Center for Disease Control and Prevention. JAMA. 2020;323(13):1239. doi:10.1001/ jama.2020.2648

5. Garcia S, Albaghdadi MS, Meraj PM, et al. Reduction in ST-segment elevation cardiac catheterization laboratory activations in the United States during COVID-19 pandemic. J Am Coll Cardiol. 2020;75 (22):2871-2872. doi:10.1016/j.jacc.2020.04.011

6. Tam CF, Cheung KS, Lam S, et al. Impact of Coronavirus Disease 2019 (COVID-19) outbreak on ST-segment-elevation myocardial infarction care in Hong Kong, China. Circ Cardiovasc Qual Outcomes. 2020;13(4):e6631. doi:10.1161/CIRCOUTCOMES.120. 006631

7. Hammad TA, Parikh M, Tashtish N, et al. Impact of COVID-19 pandemic on ST-elevation myocardial infarction in a non-COVID19 epicenter. Catheter Cardiovasc Interv. 2020. doi:10.1002/ ccd. 28997

8. Xiang D, Xiang X, Zhang W, et al. Management and outcomes of patients with STEMI during the COVID-19 pandemic in China. $J$ Am Coll Cardiol. 2020;76(11):1318-1324. PMID: 32828614; PMCID: PMC7438071. doi:10.1016/j.jacc.2020.06.039

9. Cao X, Spratt JC, Jin Z. Coronavirus disease 2019 (COVID-19) and acute cardiovascular disease management: a Chinese perspective on striking the balance. Resuscitation. 2020;152:36-38. doi:10.1016/j. resuscitation.2020.05.010

10. De Rosa S, Spaccarotella C, Basso C, et al.; Società Italiana di Cardiologia and the CCU Academy investigators group. Reduction of hospitalizations for myocardial infarction in Italy in the COVID-19 era. Eur Heart J. 2020;41(22):2083-2088. Erratum in: Eur Heart J. 2021 Feb 11;42(6):683. Erratum in: Eur Heart J. 2021 Jan 21;42(4):322. PMID: 32412631; PMCID: PMC7239145. doi:10.1093/eurheartj/ehaa409

11. Cosentino N, Bartorelli AL, Marenzi G. Time to treatment still matters in ST-elevation myocardial infarction: a call to maintain treatment effectiveness during the COVID-19 pandemic. Eur Heart $J$ Cardiovasc Pharmacother. 2020;6 (6):408-409. PMID: 32428204; PMCID: PMC7314092. doi:10.10 93/ehjcvp/pvaa054

12. Cosentino N, Assanelli E, Merlino L, Mazza M, Bartorelli AL, Marenzi G. An in-hospital pathway for acute coronary syndrome patients during the COVID-19 outbreak: initial experience under real-world suboptimal conditions. Can $J$ Cardiol. 2020;36 (6):961-964. PMID: 32376346; PMCID: PMC7162765. doi:10. 1016/j.cjca.2020.04.011
International Journal of General Medicine

\section{Publish your work in this journal}

The International Journal of General Medicine is an international, peer-reviewed open-access journal that focuses on general and internal medicine, pathogenesis, epidemiology, diagnosis, monitoring and treatment protocols. The journal is characterized by the rapid reporting of reviews, original research and clinical studies across all disease areas. The manuscript management system is completely online and includes a very quick and fair peer-review system, which is all easy to use. Visit http://www.dovepress.com/ testimonials.php to read real quotes from published authors. 